\title{
Electron paramagnetic resonance g-tensors from state interaction spin-orbit coupling density matrix renormalization group
}

Elvira R. Sayfutyarova, and Garnet Kin-Lic Chan

Citation: The Journal of Chemical Physics 148, 184103 (2018); doi: 10.1063/1.5020079

View online: https://doi.org/10.1063/1.5020079

View Table of Contents: http://aip.scitation.org/toc/jcp/148/18

Published by the American Institute of Physics 


\title{
Electron paramagnetic resonance g-tensors from state interaction spin-orbit coupling density matrix renormalization group
}

\author{
Elvira R. Sayfutyarova ${ }^{1,2}$ and Garnet Kin-Lic Chan ${ }^{2}$ \\ ${ }^{1}$ Department of Chemistry, Princeton University, Princeton, New Jersey 08543, USA \\ ${ }^{2}$ Division of Chemistry and Chemical Engineering, California Institute of Technology, \\ Pasadena, California 91125, USA
}

(Received 19 December 2017; accepted 9 April 2018; published online 8 May 2018)

\begin{abstract}
We present a state interaction spin-orbit coupling method to calculate electron paramagnetic resonance $g$-tensors from density matrix renormalization group wavefunctions. We apply the technique to compute $g$-tensors for the $\mathrm{TiF}_{3}$ and $\mathrm{CuCl}_{4}^{2-}$ complexes, a [2Fe-2S] model of the active center of ferredoxins, and a $\mathrm{Mn}_{4} \mathrm{CaO}_{5}$ model of the $\mathrm{S} 2$ state of the oxygen evolving complex. These calculations raise the prospects of determining $g$-tensors in multireference calculations with a large number of open shells. Published by AIP Publishing. https://doi.org/10.1063/1.5020079
\end{abstract}

\section{INTRODUCTION}

Electron paramagnetic resonance (EPR) is a central tool in the study of open-shell electronic structures as found in many complex transition metal systems, such as in the active sites of metalloenzymes. The primary quantity measured is the transition energy between unpaired electron states split by the external magnetic field. The proportionality between the effective magnetic moment of the unpaired electron and its spin, namely, the $g$-tensor, reports on the electronic environment of the electron. The utility of EPR arises from the high sensitivity of the $g$-tensor, which thus yields invaluable information on the nuclear geometry and electronic structure.

The most common route to compute EPR g-tensors is through density functional theory (DFT). ${ }^{1-11} \mathrm{Ab}$ initio wavefunction methods to compute $g$-tensors have also been explored, using the multireference configuration interaction, ${ }^{12-20}$ the complete active space self-consistent field (CASSCF), ${ }^{21-24}$ multireference perturbation theory, ${ }^{16,22,25}$ and coupled-cluster theory. ${ }^{22,26} \mathrm{~A}$ drawback of the electronic structure methods listed above is that they are severely limited in terms of the number of open shells they can reliably handle, restricting the kinds of transition metal complexes that can be studied. Recently, in Ref. 27, Roemelt presented an approach to compute $g$-tensors using a density matrix renormalization group (DMRG) description of the electronic structure, which is capable of treating a significantly larger number of open shells than other techniques. Here, we describe a related DMRG implementation to obtain $g$-tensors based on a state interaction spin-orbit (SISO) coupling DMRG formalism. ${ }^{27-29}$ The main methodological difference between our approach and that of Roemelt that we use is a more flexible representation of the interacting states. Both approaches include spin-orbit coupling (SOC) in the determination of the zeroth order wavefunction (i.e., they are first-order approaches), ${ }^{22-24,30}$ rather than treating it together with the magnetic field (second-order approaches). ${ }^{2-5,11-18,21,25}$ Together with Ref. 27, our work is a step toward obtaining $g$-tensors in transition metal complexes including a rigorous treatment of a large number of open shells. We first describe the formalism in Sec. II and then proceed to demonstrate the calculation of $g$-tensors at the DMRG level for the $\mathrm{TiF}_{3}$ and $\mathrm{CuCl}_{4}^{2-}$ complexes, a [2Fe-2S] model complex, and a $\mathrm{Mn}_{4} \mathrm{CaO}_{5}$ model of the $\mathrm{S} 2$ state of the oxygen evolving complex (OEC).

\section{THEORY}

We first recapitulate how to extract $g$-tensors from the spin and ab initio electronic Hamiltonians in Secs. II A and II B. Other useful presentations that we draw on can be found in Refs. 22, 24, 25, and 30. In Sec. II C, we summarize how to treat spin-orbit coupling within the spin-orbit mean-field (SOMF) approximation. Section II C also describes how to calculate all the required quantities with DMRG wavefunctions and a stateinteraction formalism, as used in our earlier work in Ref. 28.

\section{A. The spin Hamiltonian and ab initio Hamiltonian}

The relationship between theory and actual EPR spectra is provided by the effective spin Hamiltonian. The $g$-tensor arises as a parameter of the effective spin Hamiltonian and it describes the Zeeman interaction between an external magnetic field $\mathbf{B}$ and an effective spin $\mathbf{S}_{\mathrm{eff}}$ of the molecule. Other parameters of the effective spin Hamiltonian, the zero-field splitting tensor $\mathbf{D}$ and the hyperfine coupling tensor $\mathbf{A}$, define the splitting of energy levels in the absence of an external magnetic field.

Experimentally, the parameters $\mathbf{g}, \mathbf{D}$, and $\mathbf{A}$ can all be extracted from the EPR spectra. To derive them from quantum chemistry calculations, one should, first, assume one-to-one correspondence between the spin-multiplet of an effective spin Hamiltonian and ab initio many-electron wavefunctions, describing actual electronic spin states of the paramagnetic complex. The effective spin Hamiltonian in a magnetic field B is

$$
\hat{H}_{\mathrm{spin}}=\mu_{B} \mathbf{B} \cdot \mathbf{g} \cdot \hat{\mathbf{S}}_{\mathrm{eff}}+\hat{\mathbf{S}}_{\mathrm{eff}} \cdot \mathbf{D} \cdot \hat{\mathbf{S}}_{\mathrm{eff}}+\sum_{R} \hat{\mathbf{S}}_{\mathrm{eff}} \cdot \mathbf{A} \cdot \hat{\mathbf{I}}_{R}
$$

where $\mu_{B}$ is the Bohr magneton, $\hat{\mathbf{S}}_{\text {eff }}$ is the effective spin operator, and $\hat{\mathbf{I}}_{R}$ is the $R$ th nuclear spin operator. The parameters $\mathbf{g}$, $\mathbf{D}$, and $\mathbf{A}$ can be obtained by fitting the spectrum of Eq. (1) to 
the theoretical spectrum of an ab initio electronic Hamiltonian $\hat{H}$ in a magnetic field, which can be expressed as

$$
\hat{H}=\hat{H}_{0}+\hat{H}_{\mathrm{Ze}},
$$

where $\hat{H}_{0}$ is the electronic Hamiltonian in the absence of the field and $\hat{H}_{\mathrm{Ze}}$ (the Zeeman interaction) groups together all terms with an explicit dependence on the field. The Zeeman interaction can be expressed as

$$
\hat{H}_{Z e}=\mu_{B}\left(\hat{\mathbf{L}}+g_{e} \hat{\mathbf{S}}\right) \cdot \mathbf{B},
$$

where $\hat{\mathbf{L}}$ and $\hat{\mathbf{S}}$ are the total orbital and spin angular momentum operators and $g_{e} \approx 2.002319$ is the Landé factor for a free electron.

The electronic Hamiltonian $\hat{H}_{0}$ incorporates both spinindependent and spin-dependent relativistic terms. The latter include the spin-orbit coupling (SOC), spin-spin coupling, and hyperfine interactions. In this work, we determine $\mathbf{g}$ from an $a b$ initio calculation. The $g$-tensor is predominantly determined by the spin-orbit coupling which changes the effective spin of the unpaired electrons. Therefore we omit hyperfine interaction and spin-spin coupling (required for $\mathbf{D}$ and $\mathbf{A}$ ) in the expression for $\hat{H}_{0}$ and write it in the form of

$$
\hat{H}_{0}=\hat{H}_{\mathrm{SR}}+\hat{H}_{\mathrm{SOC}} .
$$

We discuss the treatment of spin-orbit coupling further in Sec. II C.

\section{B. Extracting the g-tensor}

We next consider how to extract the $g$-tensor by relating the contributions of the Zeeman interaction (3) in the ab initio Hamiltonian to that of $\mu_{B} \mathbf{B} \cdot \mathbf{g} \cdot \hat{\mathbf{S}}_{\mathrm{eff}}$ in the spin Hamiltonian (1). We follow the reasoning of Gerloch and McMeeking, first established within ligand field theory ${ }^{31}$ and later generalized to $a b$ initio Hamiltonians. ${ }^{22,25,32}$ According to Kramers theorem, in a molecular system with an odd number of electrons, i.e., with half-integer total spin, all states are at least twofold degenerate in the absence of an external magnetic field; such pairs of degenerate states are referred to as Kramers pairs and are related by time-reversal symmetry. The groundstate is then described by (at least one) Kramers pair $(\Phi, \bar{\Phi})$ at zero magnetic field. The first-order effect of an external magnetic field is to split this degenerate Kramers pair. The corresponding first-order energy can be computed from the Zeeman interaction in the ab initio Hamiltonian as well as with the spin Hamiltonian, which gives the expression for the $g$-tensor.

Using degenerate first-order perturbation theory, the splitting of the eigenvalue pair $(\Phi, \bar{\Phi})$ is obtained by diagonalizing the first-order interaction. Defining the symmetric quantity $G_{k l}=\sum_{n} g_{k n} g_{\mathrm{ln}}$, or $\mathbf{G}=\mathbf{g g}^{T}$, one can then use the formula, proposed by Gerloch and McMeeking,

$$
\mathrm{G}_{k l}=2 \sum_{u, v=\Phi, \bar{\Phi}}\left\langle u\left|\hat{L}_{k}+g_{e} \hat{S}_{k}\right| v\right\rangle\left\langle v\left|\hat{L}_{l}+g_{e} \hat{S}_{l}\right| u\right\rangle .
$$

Finally, diagonalizing $\mathbf{G}$ yields the principal axes for the $g$-tensor, and the positive square roots of the three eigenvalues are the $g$-factors $g_{1}, g_{2}, g_{3}$.
The primary ab initio task is thus to obtain the matrix elements of $\hat{L}_{k}$ and $\hat{S}_{k}$ between the degenerate Kramers groundstate pair in Eq. (5). This requires determining the eigenvalues of the electronic Hamiltonian $\hat{H}_{0}$, including the spin-orbit coupling, and computing the specific matrix elements in Eq. (5), as discussed in Sec. II C.

\section{Spin-orbit coupling and matrix elements with DMRG}

We determine spin-orbit coupled wavefunctions using the state interaction spin-orbit coupled DMRG (DMRG-SISO) that we described in Ref. 28. In this approach, the spin orbit operator $\hat{H}_{\text {So }}$ is evaluated in a basis of spin-adapted DMRG wavefunctions $\left\{\left|\Psi_{I, S, M_{S}}\right\rangle\right\}$.

It is worth briefly mentioning the different choices of spinadapted DMRG wavefunctions that can be used as the SISO basis. For a set of $N$ orbitals (sites), the DMRG wavefunction amplitudes can be written in the matrix product form. In the so-called canonical form at site $i$, this is

$$
|\Psi\rangle=\sum_{\{n\}} \mathbf{L}^{n_{1}} \ldots \mathbf{L}^{n_{i-1}} \mathbf{C}^{n_{i}} R^{n_{i+1}} \ldots \mathbf{R}^{n_{N}}\left|n_{1} \ldots n_{N}\right\rangle,
$$

where, for a given occupation string, $\mathbf{L}^{n}, \mathbf{C}^{n}, \mathbf{R}^{n}$ are $M \times M$ matrices, and the leftmost and rightmost boundary matrices are $1 \times M$ row and $M \times 1$ column vectors respectively. There are different choices to optimize the matrices $\mathbf{L}^{n}, \mathbf{C}^{n}, \mathbf{R}^{n}$ in the DMRG sweeps. In a so-called state-averaged DMRG calculation, a common renormalized basis (and thus a common set of $\mathbf{L}^{n}, \mathbf{R}^{n}$ matrices) is optimized for all the electronic states, and only the $\mathbf{C}^{n}$ matrix is unique to each state. The state-averaged DMRG representation was used in Ref. 27 by Roemelt to represent all the states (including of different spin) entering into the SISO procedure. An alternative, state-specific approach is to have different sets of $\mathbf{L}^{n}, \mathbf{C}^{n}, \mathbf{R}^{n}$ for different states in the calculations. This was the approach in our previous work, ${ }^{28}$ where we used a different set of $\mathbf{L}^{n}, \mathbf{C}^{n}, \mathbf{R}^{n}$ for states of different spins (although we used a state-averaged representation for the states of the same spin). For a given bond dimension $M$, the state-averaged approach reduces the amount of computation but results in a lower accuracy for each individual eigenstate compared to the state-specific approach. Finally, in this work, we also consider a cheaper approach, where in each spin-sector, we use the $\mathbf{L}^{n}, \mathbf{R}^{n}$ optimized only for the lowest state and represent the excited states by different $\mathbf{C}^{n}$ matrices, determined at the middle of the sweep. We denote this choice as "ground-state specific," and it has the advantage of avoiding the large number of Davidson steps required to solve for multiple states when optimizing the $\mathbf{L}^{n}, \mathbf{R}^{n}$ tensors. This allows us to compute a larger number of DMRG states to use for the SISO basis.

For the spin-orbit operator $\hat{H}_{\text {SO }}$, we use the spin-orbit mean-field (SOMF) approximation. This has been shown to approximate the effects of the full one- and two-electron BreitPauli SOC operator very accurately. ${ }^{33-36}$ In the second quantization, the one-electron SOMF Hamiltonian can be written as $^{36,37}$

$$
\hat{H}_{\mathrm{SOMF}}=\sum_{i j}\left(V_{i j}^{x} \hat{T}_{i j}^{x}+V_{i j}^{y} \hat{T}_{i j}^{y}+V_{i j}^{z} \hat{T}_{i j}^{z}\right)
$$


where $\hat{T}_{i j}^{x, y, z}$ are the Cartesian triplet excitation operators, ${ }^{38,39}$

$$
\begin{aligned}
\hat{T}_{i j}^{x} & =\frac{1}{2}\left(a_{i \alpha}^{\dagger} a_{j \beta}+a_{i \beta}^{\dagger} a_{j \alpha}\right), \\
\hat{T}_{i j}^{y} & =\frac{1}{2 i}\left(a_{i \alpha}^{\dagger} a_{j \beta}-a_{i \beta}^{\dagger} a_{j \alpha}\right), \\
\hat{T}_{i j}^{z} & =\frac{1}{2}\left(a_{i \alpha}^{\dagger} a_{j \alpha}-a_{i \beta}^{\dagger} a_{j \beta}\right),
\end{aligned}
$$

and $V_{i j}^{x, y, z}$ is an effective set of one-electron integrals, obtained as

$$
\begin{aligned}
\langle i|\hat{\mathbf{V}}| j\rangle= & \left\langle i\left|\hat{\mathbf{h}}_{1}\right| j\right\rangle+\sum_{k l} D_{k l}\left\{\left\langle i k\left|\hat{\mathbf{g}}_{12}\right| j l\right\rangle-\frac{3}{2}\left\langle i k\left|\hat{\mathbf{g}}_{12}\right| l j\right\rangle\right. \\
& \left.-\frac{3}{2}\left\langle k i\left|\hat{\mathbf{g}}_{12}\right| j l\right\rangle\right\},
\end{aligned}
$$

where $D_{k l}$ is the single-particle (not necessarily idempotent) density matrix element that takes into account single occupancy due to unpaired spins; the one- and two-electron operators are

$$
\begin{aligned}
& \hat{\mathbf{h}}_{i}=\frac{\alpha^{2}}{2} \sum_{A} Z_{A} r_{i A}^{-3} \hat{\mathbf{l}}_{i A}, \\
& \hat{\mathbf{g}}_{i j}=-\frac{\alpha^{2}}{2} \hat{\mathbf{l}}_{i j} r_{i j}^{-3},
\end{aligned}
$$

where $\alpha$ is the fine structure constant, $\hat{\mathbf{r}}_{i}, \hat{\mathbf{p}}_{i}$ are the position and momentum operators of the $i$ th electron, $r_{i j}=\left|\hat{\mathbf{r}}_{i}-\hat{\mathbf{r}}_{j}\right|$, $\hat{\mathbf{i}}_{i j}=\hat{\mathbf{r}}_{i j} \times \hat{\mathbf{p}}_{i}, \hat{\mathbf{i}}_{i A}=\hat{\mathbf{r}}_{i A} \times \hat{\mathbf{p}}_{i}, r_{i A}=\left|\hat{\mathbf{r}}_{i}-\hat{\mathbf{R}}_{A}\right|$, and $Z_{A}$ denotes the nuclear charge of the Ath nucleus. Note that this form of the SOMF operator is only valid for doublet ground states; for $S>1 / 2$, there is an additional correction $\frac{1}{2} \sum_{m n} D_{m n}\left\langle i m\left|\hat{\mathbf{g}}_{12}\right| j n\right\rangle$, where $m, n$ are the singly occupied orbitals. ${ }^{40}$ However, we have not considered this correction here as all our ground states are doublets. As we work with a spin-adapted basis, internally we do not use the Cartesian triplet operators, but rather the spherical tensor triplet operators. These are related to the Cartesian triplet operators through the linear transformation, ${ }^{38}$

$$
\begin{aligned}
\hat{T}_{i j}^{x} & =\frac{\hat{T}_{i j}^{1,-1}-\hat{T}_{i j}^{1,1}}{2}, \\
\hat{T}_{i j}^{y} & =\frac{\hat{T}_{i j}^{1,-1}+\hat{T}_{i j}^{1,1}}{2 i}, \\
\hat{T}_{i j}^{z} & =\frac{1}{\sqrt{2}} \hat{T}_{i j}^{1,0} .
\end{aligned}
$$

Using this form of the spin-orbit operator, we evaluate the Hamiltonian in Eq. (4) in the basis of spin-adapted DMRG states using the transition density matrix algorithm described in Ref. 28. Diagonalizing this yields the spin-coupled Kramers pair wavefunctions and energies.

Additionally, to determine the $\mathbf{G}$ matrix and $g$-tensors following the procedure in Sec. II B, we need the matrix representations of the operators $\hat{L}_{x}, \hat{L}_{y}, \hat{L}_{z}$ and $\hat{S}_{x}, \hat{S}_{y}, \hat{S}_{z}$ for the ground-state Kramers pair. We can obtain these from the matrix elements in the basis of spin-adapted DMRG wavefunctions $\left\{\left|\Psi_{I, S M_{S}}\right\rangle\right\}$,

$$
\left\langle\Psi_{I, S^{\prime} M_{S}^{\prime}}\left|\hat{S}_{k}\right| \Psi_{J, S M_{S}}\right\rangle=\sum_{p}\left\langle\Psi_{I, S^{\prime} M_{S}^{\prime}}\left|\hat{T}_{p p}^{k}\right| \Psi_{J, S M_{S}}\right\rangle, \quad k=x, y, z
$$

$$
\left\langle\Psi_{I, S^{\prime} M_{S}^{\prime}}\left|\hat{L}_{k}\right| \Psi_{J, S M_{S}}\right\rangle=\sum_{i j}\left\langle\Psi_{I, S^{\prime} M_{S}^{\prime}}\left|\hat{T}_{i j}^{0,0}\right| \Psi_{J, S M_{S}}\right\rangle \delta_{S S^{\prime}} \delta_{M_{S} M_{S}^{\prime}} L_{i j},
$$

where $\hat{T}_{i j}^{0,0}=\frac{1}{\sqrt{2}}\left(a_{i \alpha}^{\dagger} a_{j \alpha}+a_{i \beta}^{\dagger} a_{j \beta}\right)$ is the singlet operator and $L_{i j}=\left\langle i\left|\left(\hat{\mathbf{r}}-\hat{\mathbf{R}}_{0}\right) \times \hat{\mathbf{p}}\right| j\right\rangle$ is the orbital angular momentum integral between the molecular orbitals $i$ and $j$, calculated with respect to an arbitrary gauge origin $\mathbf{R}_{0}$, chosen here to be the coordinates of the transition metal centers. The singlet operator matrix element can be computed following Ref. 41. Once these matrix representations are obtained, they are contracted with the expansions of the Kramers pairs in the spin-adapted basis $\left\{\left|\Psi_{I, S M_{S}}\right\rangle\right\}$ to obtain representations in the Kramers basis.

\section{RESULTS AND DISCUSSION}

We implemented the above method as a stand-alone code and as a module within a development version of PySCF. ${ }^{42}$ Spin-orbit integrals in Eqs. (12) and (13) were computed using PYSCF. Additional CASSCF calculations were carried out using the Molpro package, and geometry optimization of the [2Fe-2S] complex was carried out using ORCA.

All three components of the $g$-tensor (transformed to the principal axes, denoted arbitrarily as $x, y, z$ ) can be expressed as shifts from the Landé $g$-factor

$$
g_{k}=g_{e}+\Delta g_{k}, \quad k=x, y, z,
$$

in systems with axial symmetry $g_{x}=g_{y}=g_{\perp}$ and $g_{z}=g_{\|}$. For some systems, we present $\Delta g_{k}$ shifts instead of the full $g_{k}$-values.

\section{A. $\mathrm{TiF}_{3}$}

We begin by considering the $\mathrm{TiF}_{3}$ complex. This has been widely used as a benchmark system for $g$-tensor calculations. We used ANO-RCC ${ }^{72,73}$ basis sets contracted as [4s3p2d1f] for F and [7s6p5d3f2g $1 \mathrm{~h}$ ] for Ti and a $D_{3 h}$ symmetric complex with a Ti-F bond distance of $1.774 \AA$, following Ref. 25 ; the calculations employed the $C_{s}$ subgroup, with the Ti atom at the origin, one $\mathrm{F}$ atom on the $\mathrm{y}$ axis, and the other two in the xy plane.

A minimal active space for this complex is formed by the $3 d$ orbitals of Ti, giving a $(1 \mathrm{e}, 5 \mathrm{o})$ active space. However, there is no correlation within this space. To construct a larger active space, we further included the $2 s, 2 p$ orbitals of each $\mathrm{F}$ atom and the $3 s, 3 p, 4 s, 4 p$ orbitals of Ti. This gives a (33e, 25o) active space that includes the dominant core-valence and valence-virtual correlation effects, both for the energies and the density matrices.

The ground ${ }^{2} A_{1}^{\prime}$ state has one unpaired electron in the $3 d_{z^{2}}$ orbital. The lowest excited states are metal centered ligand-field states; charge-transfer states have been seen to give negligible contributions to the $g$-values in previous studies. ${ }^{25}$ Thus we choose the lowest 5 ligand-field states for the stateinteraction basis. We first optimized $3 d$ orbitals using stateaveraged CASSCF in the (1e,5o) active space using MolPRo, with scalar relativistic effects included with the second-order 
TABLE I. The electronic states of $\mathrm{TiF}_{3}\left(\mathrm{~cm}^{-1}\right)$ from this work, experiment, and previous theoretical studies.

\begin{tabular}{lrcccccc}
\hline \hline State & $\begin{array}{c}\text { CASSCF } \\
(1 \mathrm{e}, 5 \mathrm{o})\end{array}$ & $\begin{array}{c}\text { DMRG } \\
(33 \mathrm{e}, 25 \mathrm{o})\end{array}$ & $\begin{array}{c}\text { MRCISD+Q } \\
(1 \mathrm{e}, 5 \mathrm{o})\end{array}$ & $\mathrm{CCSD}(\mathrm{T})^{46}$ & $\mathrm{CCSD}(\mathrm{T})^{22}$ & $\begin{array}{c}\mathrm{CASPT2}^{22} \\
(7 \mathrm{e}, 8 \mathrm{o})\end{array}$ & $\begin{array}{c}\mathrm{CASPT2}^{25} \\
(17 \mathrm{e}, 13 \mathrm{o})\end{array}$ \\
\hline$X^{2} A_{1}^{\prime}$ & & & & & & & \\
$1^{2} E^{\prime \prime}$ & 3502 & 5785 & 4414 & 5181 & 5600 & 3700 & 4789 \\
$1^{2} E^{\prime}$ & 20158 & 22538 & 19379 & 19855 & & & \\
\hline \hline
\end{tabular}

Douglas-Kroll approximation. ${ }^{43-45}$ We then used DMRG in a larger $(33 \mathrm{e}, 25 \mathrm{o})$ active space (using the $(1 \mathrm{e}, 5 \mathrm{o}) \mathrm{CASSCF}$ orbitals). The DMRG energy for each state was converged to better than $10^{-6} E_{h}$ with a bond-dimension of $M=3000$.

The 5 lowest electronic states, without SOC, are presented in Table I. The calculated and reference $g$-tensors from the literature are summarized in Table II.

The $(1 \mathrm{e}, 5 \mathrm{o}) \mathrm{CASSCF}$ calculation underestimates the energy of the lowest excited $1^{2} E^{\prime \prime}$ state compared to larger active space calculations. The CASPT2 (complete active space second-order perturbation theory) energies from Ref. 22, obtained with the $(7 \mathrm{e}, 8 \mathrm{o})$ active space [obtained from the $(1 \mathrm{e}, 5 \mathrm{o})$ active space by including three additional occupied ligand orbitals], confirm this observation. The DMRG (33e,25o) energies are in good agreement with the literature $\operatorname{CCSD}(\mathrm{T})$ (coupled cluster singles, doubles with perturbative triples corrections) energies. Including SOC removes the degeneracy of the $1^{2} E^{\prime \prime}$ and $1^{2} E^{\prime}$ states and results in $144 \mathrm{~cm}^{-1}$ and $228 \mathrm{~cm}^{-1}$ splittings at the DMRG level, respectively, giving two pairs of states with excitation energies $5719 \mathrm{~cm}^{-1}, 5863 \mathrm{~cm}^{-1}$ and 22 $428 \mathrm{~cm}^{-1}, 22656 \mathrm{~cm}^{-1}$.

For the $g$-tensor, the DFT-based approaches significantly underestimate $\Delta g_{\perp}{ }^{1,4,5,19}$ The wavefunction-based $g$-shifts, including from the $(33 \mathrm{e}, 25 \mathrm{o})$ DMRG-SISO calculation, are all in quite good agreement with experiment; there is particularly close agreement between the DMRG-SISO $g$-values and those obtained from $\operatorname{CCSD}(\mathrm{T})$.

\section{B. $\mathrm{CuCl}_{4}^{2-}$}

We next consider the square planar $\mathrm{CuCl}_{4}^{2-}$ complex. This can be viewed as a model complex for copper sites in blue copper proteins, such as plastocyanin. We used ANO-RCC basis sets contracted to [5s4p2d1f] for $\mathrm{Cl}$ and [7s6p5d3f2g $1 \mathrm{~h}$ ] for $\mathrm{Cu}$ and a $D_{4 h}$ symmetric complex with a $\mathrm{Cu}-\mathrm{Cl}$ distance of $2.291 \AA$ as in Ref. 25. For the active space, we considered a $\mathrm{Cu} 3 d, 4 s, 4 d(9 \mathrm{e}, 11 \mathrm{o})$ active space, a minimal active space including double-shell effects. We also considered two larger active spaces: one with additional $3 s, 3 p, 4 p \mathrm{Cu}$ orbitals and four $\sigma$-orbitals formed by the $3 d$ orbitals of $\mathrm{Cu}$ and the $3 p$ orbitals of $\mathrm{Cl}$ atoms, giving a $(25 \mathrm{e}, 22 \mathrm{o})$ active space, and one that further incorporates the $3 p$ orbitals of the $\mathrm{Cl}$ atoms that provide $\pi$-interactions with the metal, giving a (41e, 30o) active space.

We first optimized the $3 d$ orbitals using state-averaged CASSCF with the $(9 \mathrm{e}, 11 \mathrm{o})$ active space using Molpro. ${ }^{48}$ Scalar relativistic effects were included using the secondorder Douglas-Kroll approximation. ${ }^{43-45}$ DMRG calculations were then performed with the $(25 \mathrm{e}, 22 \mathrm{o})$ and $(41 \mathrm{e}, 30 \mathrm{o})$ active spaces for the lowest 5 ligand-field excited states. These 5 states were used as the SISO basis. The DMRG energy for each state was converged to better than $10^{-6} E_{h}$ accuracy using a bond-dimension of $M=3000$. The electronic states with and without SOC are presented in Table III. Calculated and reference g-tensors from the literature are summarized in Table IV.

The $(9 e, 110)$ active space significantly underestimates the excitation energies of all the states. Including the near-valence orbitals of $\mathrm{Cu}$ and the $3 p$ orbitals of $\mathrm{Cl}$ atoms in the DMRG calculation recovers an important piece of the dynamic electron correlation, shifting the excitation energies upwards by $\approx 2300$ $2700 \mathrm{~cm}^{-1}$. To verify the effects of dynamic correlation, we have also carried out DMRG-NEVPT2 calculations (Nelectron valence state perturbation theory based on a DMRG reference wavefunction) for each state $;^{53}$ for the corresponding $g$-tensor calculations, the energies were used to shift the SISO matrix elements by $\Delta \hat{H}_{\mathrm{SR}}^{I J}=0.5\left(\Delta E_{P T 2}^{I}+\Delta E_{P T 2}^{J}\right)\left\langle\Psi_{I} \mid \Psi_{J}\right\rangle .{ }^{37}$ The DMRG-NEVPT2 excitation energies are shifted further upwards, giving improved agreement with the experimental excitation energies (see Table IV). Including the SOC in the DMRG-SISO has a large effect on the $1^{2} A_{1 g}$ excitation, although it remains below the experimental number.

TABLE II. Calculated and experimental $\Delta g$ shifts for $\mathrm{TiF}_{3}$ (in ppt).

\begin{tabular}{|c|c|c|c|c|c|c|c|c|c|}
\hline g-values & $\begin{array}{c}\text { DMRG-SISO } \\
(33 \mathrm{e}, 25 \mathrm{o})\end{array}$ & $\begin{array}{c}\text { CASPT2 }^{22} \\
(7 \mathrm{e}, 8 \mathrm{o})\end{array}$ & $\operatorname{CCSD}(\mathrm{T})^{22}$ & $\begin{array}{c}\text { CASPT2 }^{25} \\
(17 \mathrm{e}, 13 \mathrm{o})\end{array}$ & $\mathrm{MRCI}^{17}$ & $\mathrm{SORCI}^{16}$ & ZORA $^{1}$ & BP86 & Expt. $^{47}$ \\
\hline$\Delta g_{\perp}$ & -113.7 & -125.3 & -118 & $\begin{array}{l}-143.5 \text { (I) } \\
-147.6 \text { (II) }\end{array}$ & -115.3 & -75.5 & -79.7 & $\begin{array}{l}-30.7^{19} \\
-36.0^{5} \\
-26.6^{4}\end{array}$ & $\begin{array}{l}-111.3^{\mathrm{a}} \\
-123.7^{\mathrm{b}}\end{array}$ \\
\hline$\Delta g_{\|}$ & -1.6 & -2.4 & -1.6 & $\begin{array}{c}0.0 \text { (I) } \\
-2.5 \text { (II) }\end{array}$ & -0.9 & -0.1 & -1.1 & $\begin{array}{l}-0.9^{19} \\
-1.3^{5} \\
-1.1^{4}\end{array}$ & $\begin{array}{l}-11.1^{\mathrm{a}} \\
-3.7^{\mathrm{b}}\end{array}$ \\
\hline
\end{tabular}

${ }^{\mathrm{a}}$ From the EPR spectrum of $\mathrm{TiF}_{3}$ in solid neon at $4 \mathrm{~K}$.

${ }^{\mathrm{b}}$ From the EPR spectrum of $\mathrm{TiF}_{3}$ in solid argon at $4 \mathrm{~K}$. 
TABLE III. The electronic states of $\mathrm{CuCl}_{4}^{2-}\left(\mathrm{cm}^{-1}\right)$. The SOC-corrected energies from DMRG-SISO are given in parentheses.

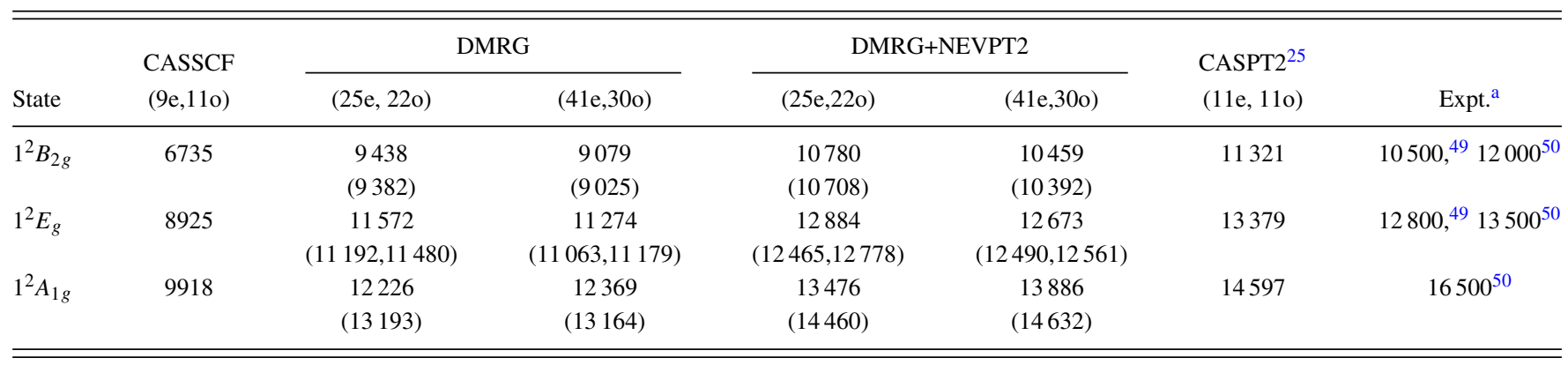

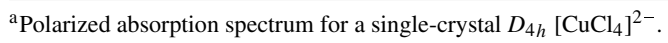

TABLE IV. Calculated and experimental $\Delta g_{\perp}$ and $\Delta g_{\|}$shifts of $\mathrm{CuCl}_{4}^{2-}$ (ppt).

\begin{tabular}{|c|c|c|c|c|c|c|c|}
\hline \multirow[b]{2}{*}{ g-values } & \multicolumn{2}{|c|}{ DMRG-SISO } & \multicolumn{2}{|c|}{ DMRG-SISO+NEVPT2 } & \multirow[b]{2}{*}{$\mathrm{LFT}^{\mathrm{a} 51}$} & \multirow{2}{*}{$\begin{array}{l}\text { CASPT2 }^{25} \\
(11 \mathrm{e}, 11 \mathrm{o})\end{array}$} & \multirow[b]{2}{*}{ Expt. } \\
\hline & $(25 \mathrm{e}, 22 \mathrm{o})$ & $(41 \mathrm{e}, 30 \mathrm{o})$ & $(25 \mathrm{e}, 22 \mathrm{o})$ & $(41 \mathrm{e}, 30 \mathrm{o})$ & & & \\
\hline$\Delta g_{\perp}$ & 100.6 & 82.6 & 92.3 & 77.1 & 117 & 96.1 (I); 77.7 (II) & $47,,^{52} 38^{50}$ \\
\hline$\Delta g_{\|}$ & 517.9 & 529.8 & 458.7 & 464.3 & 531 & 466.0 (I);437.7 (II) & $230,,^{52} 219^{50}$ \\
\hline
\end{tabular}

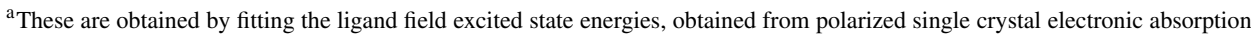
spectroscopy, to the $g$-tensor expression in the ligand field approximation.

The $g$-values calculated with the different theoretical methods are roughly comparable. In the DMRG-SISO calculations, the effect of increasing the active space size or including dynamic correlation is to lower $\Delta g_{\perp}$ but raise $\Delta g_{\|}$. However, the DMRG-SISO $g$-values remain too large when compared with experiment, almost by a factor of 2 . This is consistent with earlier CASPT2 calculations ${ }^{25}$ which also found an overestimation by a factor of 2 . In Ref. 25 , it is argued that as the CASPT2 excitation energies are quite accurate for this compound, and that the error must arise in the density matrices, which yield too large matrix elements for the spin-orbit coupling operator due to too much ionic character in the $\mathrm{Cu}-\mathrm{Cl}$ bond. Our results indicate that this remains true even when the density matrices are relaxed in the larger active space treated by DMRG.

\section{C. $[2 \mathrm{Fe}-2 \mathrm{~S}]^{+}$}

We now consider the $\left[\mathrm{Fe}_{2} \mathrm{~S}_{2}\left(\mathrm{SCH}_{3}\right)_{4}\right]^{3-}$ complex. This can be considered to be a model of the active site in certain iron-sulfur proteins, such as the ferredoxins in their reduced form. To assess geometrical effects, we performed calculations at three different geometries: the relaxed geometry from Ref. 54 (geometry I), which was optimized by the authors at the def2-SVP/BP86 level of theory, and two geometries, which we optimized at the def2-TZVP/TPSSh level of theory with (geometry II) and without inclusion of solvation effects (geometry III), using ORCA. ${ }^{55}$ Solvation was included via the COnductor-like Screening Model (COSMO) model with a dielectric constant of 4.0 which crudely imitates a protein environment. Table $\mathrm{V}$ summarizes the structural parameters for the three geometries used for the model $\left[\mathrm{Fe}_{2} \mathrm{~S}_{2}\left(\mathrm{SCH}_{3}\right)_{4}\right]^{3-}$ complex as well as the geometries of two high-resolution X-ray structures of two reduced ferredoxin species: from the green alga Chlorella fusca ${ }^{56}$ and from the cyanobacterium Anabaena PCC $7119 .{ }^{57}$ As one can see, geometry II, obtained by including solvation effects, mimics the ferredoxin active center better than the other model geometries.

To determine a suitable active space at each geometry, we first carried out an unrestricted Kohn-Sham (UKS)

TABLE V. Structural parameters for the reduced [ $2 \mathrm{Fe}-2 \mathrm{~S}]$ cluster, obtained from optimized geometries of a model compound $\left[\mathrm{Fe}_{2} \mathrm{~S}_{2}\left(\mathrm{SCH}_{3}\right)_{4}\right]^{3-}$ and high-resolution X-ray crystal structures of different reduced ferredoxins.

\begin{tabular}{|c|c|c|c|c|c|}
\hline \multirow[b]{2}{*}{ Bond lengths, angles } & \multicolumn{3}{|c|}{ Optimized geometries } & \multicolumn{2}{|c|}{$\mathrm{X}$-ray structures from } \\
\hline & I & II & III & Chlorella fusca ${ }^{56}$ & Anabaena PCC $7119^{57}$ \\
\hline $\mathrm{Fe} 1-\mathrm{Fe} 2(\AA)$ & 2.914 & 2.827 & 2.775 & 2.733 & 2.749 \\
\hline Fe1-S1 (̊̊) & 2.365 & 2.355 & 2.326 & 2.230 & 2.293 \\
\hline $\mathrm{Fe} 2-\mathrm{S} 1(\AA)$ & 2.267 & 2.214 & 2.222 & 2.196 & 2.235 \\
\hline $\mathrm{Fe} 1-\mathrm{S} 2(\AA)$ & 2.379 & 2.357 & 2.336 & 2.224 & 2.261 \\
\hline $\mathrm{Fe} 2-\mathrm{S} 2(\AA)$ & 2.260 & 2.178 & 2.213 & 2.157 & 2.178 \\
\hline Fe1-S1-Fe2 (deg) & 77.9 & 76.4 & 75.2 & 76.3 & 74.7 \\
\hline Fe1-S2-Fe2 (deg) & 77.8 & 76.3 & 75.1 & 77.2 & 76.5 \\
\hline S1-Fe1-S2 (deg) & 98.8 & 99.2 & 101.1 & 101.4 & 101.8 \\
\hline S1-Fe2-S2 (deg) & 105.5 & 108.1 & 108.5 & 104.8 & 106.4 \\
\hline
\end{tabular}


TABLE VI. Dependence of $g$-values of the reduced [2Fe-2S] complex on the number of electronic states included in the doublet and quartet manifolds (for geometry I). The DMRG energies were converged using $M=3000$.

\begin{tabular}{llcc}
\hline \hline & \multicolumn{3}{c}{ No. of doublet and quartet states in DMRG-SISO* } \\
\cline { 2 - 4 }$g$-values & $2+2$ & $3+3$ & $5+5$ \\
\hline$g_{x}$ & 1.989 & 1.888 & 1.807 \\
$g_{y}$ & 2.004 & 1.989 & 1.931 \\
$g_{z}$ & 2.006 & 2.004 & 1.969 \\
\hline \hline
\end{tabular}

BP86/TZP-DKH calculation of the high spin state with $S=9 / 2$. Scalar relativistic effects were included using the exact-twocomponent (X2C) approach ${ }^{58}$ implemented in PySCF. From the alpha and beta UKS orbitals, we constructed unrestricted natural orbitals (UNOs). From the UNO occupations, the orbitals were separated into three subspaces: doubly occupied, singly occupied, and virtual molecular orbitals. Next, localized orbitals were constructed by projecting atomic orbitals into these 3 spaces (e.g., a localized core 1 s orbital is obtained by projecting a $1 \mathrm{~s}$ orbital into the doubly occupied space), followed by a subsequent orthonormalization within the spaces. By population analysis and visualization of the projected AO's, we determined a suitable active space. In this way, we obtained a $(31 \mathrm{e}, 360)$ active space including the (1) $3 d, 3 d^{\prime}, 4 s$ orbitals for $\mathrm{Fe}$, (2) three $3 p$ and two lowest-energy $3 d$ orbitals on each bridging $\mathrm{S}$ atom, and (3) an additional $3 p$ orbital on each ligand $\mathrm{S}$ atom.

We carried out DMRG calculations for the doublet, quartet, and hextet states, i.e., with $S=1 / 2,3 / 2,5 / 2$. Note that sextet states and higher do not directly spin orbit couple with the ground doublet state; however, they can contribute indirectly to the $g$-tensor via coupling with lower spin states, changing their energies. Sharma et al. ${ }^{54}$ have shown that there are a large number of spin states at low energies in these systems; thus, we can expect a large number of states to contribute in the DMRGSISO procedure. Table VI shows how $g$-values change with the number of doublet, quartet, and hextet states included in the DMRG-SISO calculations for geometry I. Table VIII presents the $g$-values obtained for all three geometries using the 5 lowest doublet and 5 lowest quartet states (10 states in total). To include even more states in the DMRG-SISO, we used the "ground-state specific" procedure described in Sec. II C to compute a large number of excited states without explicitly reoptimizing their renormalized bases. Using this approach, we were able to include up to 10 doublet and 10 quartet states. The effect of including more states on the $g$-tensor for geometry II is presented in Table VII. We see that after 10 doublet and 10 quartet states, the $g$-tensor appears well converged; the remaining uncertainty is on the $O(0.01)$ level.

Comparing the $g$-tensors from the model DMRG-SISO calculations and the experimental $g$-tensors in biological complexes in Table VIII, we find that while the middle $g$-value is in reasonable agreement with experiment, the other two $g$-values are significantly underestimated. As we have argued, we do not think this is due to the insufficient states in the DMRGSISO procedure. Further, our earlier work has suggested that the lowest spin state excitation energies are at least qualitatively reasonable in the active space. We have computed the partial charges on the Fe and S atoms in the DMRG-SISO calculation as well as with the BP86 functional (see Table IX). As can be seen, the DMRG predicts significantly more ionic $\mathrm{Fe}-\mathrm{S}$ bonds than at the DFT level. This suggests that the error in the $g$-values may once again arise from errors in the density

TABLE VII. Dependence of $g$-values of the reduced [2Fe-2S] complex on the number of electronic states included in the doublet and quartet manifolds (for geometry II). The DMRG energies were converged to better than $10^{-3} E_{h}$ accuracy using $M=3000$ for our standard state-specific procedure and using $M=3200$ for the cheap ground-state specific procedure.

\begin{tabular}{|c|c|c|c|c|c|c|}
\hline \multirow[b]{2}{*}{$g$-values } & \multicolumn{2}{|c|}{$3+3$} & \multicolumn{2}{|c|}{$5+5$} & \multicolumn{2}{|c|}{$10+10$} \\
\hline & $\begin{array}{l}\text { State- } \\
\text { specific }\end{array}$ & $\begin{array}{l}\text { Ground-state } \\
\text { specific }\end{array}$ & $\begin{array}{l}\text { State- } \\
\text { specific }\end{array}$ & $\begin{array}{l}\text { Ground-state } \\
\text { specific }\end{array}$ & $\begin{array}{l}\text { State- } \\
\text { specific }\end{array}$ & $\begin{array}{c}\text { Ground-state } \\
\text { specific }\end{array}$ \\
\hline$g_{x}$ & 1.909 & 1.907 & 1.834 & 1.831 & N/A & 1.831 \\
\hline$g_{y}$ & 1.959 & 1.953 & 1.945 & 1.935 & N/A & 1.935 \\
\hline$g_{z}$ & 2.004 & 2.004 & 1.957 & 1.962 & N/A & 1.961 \\
\hline
\end{tabular}

TABLE VIII. $g$-values of the reduced [2Fe-2S] complex from DMRG-SISO calculations for all geometries using the 5 lowest doublet and 5 lowest quartet states (10 states in total) and from experiment. The DMRG energies were converged using $M=3000$.

\begin{tabular}{|c|c|c|c|c|c|c|c|}
\hline \multirow[b]{3}{*}{$g$-values } & \multicolumn{3}{|c|}{ Theory } & \multicolumn{4}{|c|}{ Experiment } \\
\hline & \multicolumn{3}{|c|}{$\left[\mathrm{Fe}_{2} \mathrm{~S}_{2}\left(\mathrm{SCH}_{3}\right)_{4}\right]^{3-}$} & \multirow[b]{2}{*}[\mathrm{Fe}_{2}\mathrm{S}_{2}(\mathrm{SPh})_{4}]{$^{3-}$} & \multicolumn{3}{|c|}{ Reduced ferredoxin } \\
\hline & $\mathrm{I}$ & II & III & & Anabaena ${ }^{59}$ & Spinach $^{\mathrm{a}}$ & Clostridium $^{\mathrm{b}}$ \\
\hline$g_{x}$ & 1.807 & 1.834 & 1.852 & $1.87-1.91$ & 1.88 & $1.86-1.89$ & $1.89-1.93$ \\
\hline$g_{y}$ & 1.931 & 1.945 & 1.936 & $1.91-1.95$ & 1.96 & $1.94-1.96$ & $1.95-1.96$ \\
\hline$g_{z}$ & 1.969 & 1.957 & 1.964 & $2.00-2.01$ & 2.05 & $2.04-2.05$ & $2.00-2.01$ \\
\hline
\end{tabular}

${ }^{\mathrm{a}}$ The range is given based on $g$-values for spinach ferredoxin presented in Refs. 60-65.

${ }^{\mathrm{b}}$ The range is given based on $g$-values for Clostridium pasteurianum ferredoxin in Refs. 61 and 66 . 
TABLE IX. Selected Löwdin partial charges for the $\left[\mathrm{Fe}_{2} \mathrm{~S}_{2}\left(\mathrm{SCH}_{3}\right)_{4}\right]^{3-}$ complex.

\begin{tabular}{lrrrrr}
\hline \hline & \multicolumn{2}{c}{ Geometry I } & & \multicolumn{2}{c}{ Geometry II } \\
\cline { 2 - 3 } \cline { 5 - 6 } Atom & BP86 & DMRG & & BP86 & DMRG \\
\hline Fe1 & 0.705 & 1.097 & & 0.690 & 0.854 \\
Fe2 & 0.776 & 1.047 & & 0.736 & 0.994 \\
S1 & -0.782 & -0.910 & & -0.790 & -0.927 \\
S2 & -0.811 & -0.942 & & -0.801 & -0.936 \\
\hline \hline
\end{tabular}

and ionicity of the metal-ligand bonds, similar to the case of $\mathrm{CuCl}_{4}^{2-}$ above.

\section{D. $\mathrm{Mn}_{4} \mathrm{CaO}_{5}$ model of the $\mathrm{S} 2$ state of OEC}

Here we consider a model of the S2 state of the oxygen evolving complex in photosystem II. We use an oxygenbridged tetramanganese calcium $\mathrm{Mn}_{4} \mathrm{CaO}_{5}$ complex using the geometry in Ref. 67, which was optimized for the S2 state using broken-symmetry DFT at the def2-TZVP(-f)/BP86-D3 level of theory, and with the zeroth-order regular approximation (ZORA) to include scalar relativistic effects. This model has previously been studied using DMRG in Ref. 68.

We first carried out an unrestricted BP86/def2-TZVPPDKH basis set calculation on the high spin state with $S=13 / 2$ and we included scalar-relativistic effects using the X2C method. As in the previous example, from the alpha and beta UKS orbitals, we constructed UNOs which were further separated into three subspaces: doubly occupied, singly occupied, and virtual molecular orbitals. Next, we constructed localized orbitals by projecting atomic orbitals and chose the $2 p$ orbitals of the five bridging oxygens and $3 d$ orbitals of the four manganese centers to comprise the active space. With this $(43 \mathrm{e}, 35 \mathrm{o})$ active space, we calculated 7 doublet and 11 quartet states using DMRG-CI with $M=1000$. (Previous studies in Ref. 68 showed that the DMRG energy can be converged to beyond chemical accuracy at this bond dimension.) We obtained $g$-values of $2.0014484,2.0014628$, and 2.0022972 , giving (small) $g$-shifts relative to the Landé factor of $-870,-856$, and $-22 \mathrm{ppm}$. We are not aware of other theoretical estimates for these $g$-values. However, in the experimental EPR spectrum of the OEC S2 state with $S=1 / 2$, one observes a multiline EPR signal centered at $g=2.0 .^{67,69-71}$ There is evidence also that this signal is quite isotropic, ${ }^{67,69}$ and this is consistent with the nearly isotropic $g$-tensor that we compute.

\section{CONCLUSIONS}

In this work, we presented a method to calculate molecular $g$-tensors using state-interaction spin-orbit coupling and density matrix renormalization group wavefunctions. We have demonstrated this approach on two mononuclear transition metal complexes and a binuclear and tetranuclear transition metal complexes. Our results show that it is possible to converge the calculations with respect to the number of states entering in the state-interaction picture. Remaining discrepancies often appear attributable to the description of the ionic/covalent character of the metal ligand bond, which requires a careful balance between the static and dynamic correlation. Nonetheless, our work is a step toward truly multireference calculations of $g$-tensors in complex systems, including in the study of larger active sites in metalloenzymes.

\section{ACKNOWLEDGMENTS}

We acknowledge the U.S. National Science Foundation for funding this research through the Award No. NSF:CHE1657286.

${ }^{1}$ E. van Lenthe, P. E. S. Wormer, and A. van der Avoird, J. Chem. Phys. 107, 2488 (1997).

${ }^{2}$ G. Schreckenbach and T. Ziegler, J. Phys. Chem. A 101, 3388 (1997).

${ }^{3}$ S. Patchkovskii and T. Ziegler, J. Phys. Chem. A 105, 5490 (2001).

${ }^{4}$ O. L. Malkina, J. Vaara, B. Schimmelpfenning, M. Munzarova, V. Malkin, and M. Kaupp, J. Am. Chem. Soc. 122, 9206 (2000).

${ }^{5}$ M. Kaupp, R. Reviakine, O. L. Malkina, A. Arbuznikov, B. Schimmelpfennig, and V. G. Malkin, J. Comput. Chem. 23, 794 (2002).

${ }^{6}$ F. Neese, J. Chem. Phys. 115, 11080 (2001).

${ }^{7}$ K. M. Neyman, D. I. Ganyushin, A. V. Matveev, and V. A. Nasluzov, J. Phys. Chem. A 106, 5022 (2002).

${ }^{8}$ I. Malkin, O. Malkina, V. Malkin, and M. Kaupp, J. Chem. Phys. 123, 244103 (2005).

${ }^{9}$ S. Komorovský, M. Repiský, O. L. Malkina, V. G. Malkin, I. Malkin, and M. Kaupp, J. Chem. Phys. 124, 084108 (2006).

${ }^{10}$ M. Repiský, S. Komorovský, E. Malkin, O. L. Malkina, and V. G. Malkin, Chem. Phys. Lett. 488, 94 (2010).

${ }^{11}$ Z. Rinkevicius, L. Telyatnyk, P. Sałek, O. Vahtras, and H. Ågren, J. Chem. Phys. 119, 10489 (2003).

${ }^{12}$ G. Lushington, P. Bündgen, and F. Grein, Int. J. Quantum Chem. 55, 377 (1995).

${ }^{13}$ P. Bündgen, G. Lushington, and F. Grein, Int. J. Quantum Chem. 56, 283 (1995).

${ }^{14}$ G. H. Lushington and F. Grein, Theor. Chim. Acta 93, 259 (1996).

${ }^{15}$ P. J. Bruna, G. H. Lushington, and F. Grein, Chem. Phys. 225, 1 (1997).

${ }^{16}$ F. Neese, Chem. Phys. Lett. 380, 721 (2003).

${ }^{17}$ S. Brownridge, F. Grein, J. Tatchen, M. Kleinschmidt, and C. Marian, J. Chem. Phys. 118, 9552 (2003).

${ }^{18}$ F. Neese, Mol. Phys. 105, 2507 (2007).

${ }^{19}$ F. Neese, Int. J. Quantum Chem. 83, 104 (2001).

${ }^{20}$ J. Tatchen, M. Kleinschmidt, and C. Marian, J. Chem. Phys. 130, 154106 (2009).

${ }^{21}$ O. Vahtras, B. Minaev, and H. Ågren, Chem. Phys. Lett. 281, 186 (1997).

${ }^{22}$ H. Bolvin, ChemPhysChem. 7, 1575 (2006).

${ }^{23}$ D. Ganyushin and F. Neese, J. Chem. Phys. 138, 104113 (2013).

${ }^{24}$ T. N. Lan, J. Chalupský, and T. Yanai, Mol. Phys. 113, 1750 (2015).

${ }^{25}$ S. Vancoillie, P.-Å. Malmqvist, and K. Pierloot, ChemPhysChem 8, 1803 (2007).

${ }^{26}$ J. Gauss, M. Kállay, and F. Neese, J. Phys. Chem. A 113, 11541 (2009).

${ }^{27}$ M. Roemelt, J. Chem. Phys. 143, 044112 (2015).

${ }^{28}$ E. R. Sayfutyarova and G. K.-L. Chan, J. Chem. Phys. 144, 234301 (2016).

${ }^{29}$ S. Knecht, S. Keller, J. Autschbach, and M. Reiher, J. Chem. Theory Comput. 12, 5881 (2016).

${ }^{30}$ L. F. Chibotaru and L. Ungur, J. Chem. Phys. 137, 064112 (2012).

${ }^{31}$ M. Gerloch and R. F. McMeeking, J. Chem. Soc., Dalton Trans. 1975, 2443.

${ }^{32}$ L. F. Chibotaru, M. F. A. Hendrickx, S. Clima, J. Larionova, and A. Ceulemans, J. Phys. Chem. A 109, 7251 (2005).

${ }^{33}$ C. M. Marian, in Reviews in Computational Chemistry, Volume 17, edited by K. B. Lipkowitz and D. B. Boyd (Wiley-VCH, New York, 2001), pp. 99-204.

${ }^{34}$ B. A. Heß, C. M. Marian, U. Wahlgren, and O. Gropen, Chem. Phys. Lett. 251, 365 (1996).

${ }^{35}$ J. Tatchen and C. M. Marian, Chem. Phys. Lett. 313, 351 (1999).

${ }^{36}$ F. Neese, J. Chem. Phys. 122, 034107 (2005).

${ }^{37}$ P.-Å. Malmqvist, B. Roos, and B. Schimmelpfennig, Chem. Phys. Lett. 357, 230 (2002).

${ }^{38}$ T. Helgaker, P. Jørgensen, and J. Olsen, Molecular Electronic Structure Theory (Wiley, Chichester, 2000).

${ }^{39}$ T. Helgaker, S. Coriani, P. Jørgensen, K. Kristensen, J. Olsen, and K. Ruud, Chem. Rev. 112, 543 (2012). 
${ }^{40}$ A. Van Yperen-De Deyne, E. Pauwels, V. Van Speybroeck, and M. Waroquier, Phys. Chem. Chem. Phys. 14, 10690 (2012).

${ }^{41}$ S. Sharma and G. K.-L. Chan, J. Chem. Phys. 136, 124121 (2012).

${ }^{42}$ Q. Sun, T. C. Berkelbach, N. S. Blunt, G. H. Booth, S. Guo, Z. Li, J. Liu, J. McClain, E. R. Sayfutyarova, S. Sharma, S. Wouters, and G. K.-L. Chan, Wiley Interdiscip. Rev.: Comput. Mol. Sci. 8, e1340 (2017).

${ }^{43}$ A. Wolf, M. Reiher, and B. A. Hess, J. Chem. Phys. 117, 9215 (2002).

${ }^{44}$ M. Reiher and A. Wolf, J. Chem. Phys. 121, 2037 (2004).

${ }^{45}$ M. Reiher and A. Wolf, J. Chem. Phys. 121, 10945 (2004).

${ }^{46}$ V. G. Solomonik and A. A. Mukhanov, Russ. J. Phys. Chem. A 88, 85 (2014).

${ }^{47}$ T. C. DeVore and J. W. Weltner, J. Am. Chem. Soc. 99, 4700 (1977).

${ }^{48}$ H.-J. Werner, P. J. Knowles, G. Knizia, F. R. Manby, and M. Schütz, Wiley Interdiscip. Rev.: Comput. Mol. Sci. 2, 242 (2012).

${ }^{49}$ R. D. Willett, O. L. Liles, and C. Michelson, Inorg. Chem. 6, 1885 (1967).

${ }^{50}$ E. I. Solomon, R. K. Szilagyi, S. D. George, and L. Basumallick, Chem. Rev. 104, 419 (2004).

${ }^{51}$ F. Neese and E. I. Solomon, "Interpretation and calculation of spinHamiltonian parameters in transition metal complexes," in Magnetism: Molecules to Materials IV: Nanosized Magnetic Materials, edited by J. $\mathrm{S}$. Miller and M. Drillon (Wiley-VCH Verlag GmbH \& Co, Weinheim, FRG, 2002), pp. 345-466.

${ }^{52}$ C. Chow, K. Chang, and R. D. Willett, J. Chem. Phys. 59, 2629 (1973).

${ }^{53}$ S. Guo, M. A. Watson, W. Hu, Q. Sun, and G. K.-L. Chan, J. Chem. Theory Comput. 12, 1583 (2016).

${ }^{54}$ S. Sharma, K. Sivalingam, F. Neese, and G. K.-L. Chan, Nat. Chem. 6, 927 (2014).

${ }^{55}$ F. Neese, Wiley Interdiscip. Rev.: Comput. Mol. Sci. 2, 73 (2012).

${ }^{56}$ M. T. Bes, E. Parisini, L. A. Inda, L. M. Saraiva, M. L. Peleato, and G. M. Sheldrick, Structure 7, 1201 (1999).

${ }^{57}$ R. Morales, M. Chron, G. Hudry-Clergeon, Y. Petillot, S. Norager, M. Medina, and M. Frey, Biochemistry 38, 15764 (1999).
${ }^{58}$ D. Peng, N. Middendorf, F. Weigend, and M. Reiher, J. Chem. Phys. 138, 184105 (2013).

${ }^{59}$ H. Cheng, B. Xia, G. H. Reed, and J. L. Markley, Biochemistry 33, 3155 (1994).

${ }^{60}$ J. Fritz, R. Anderson, J. Fee, G. Palmer, R. H. Sands, J. C. M. Tsibris, J. C. Gunsalus, W. H. Orme-Johnson, and H. Beinert, Biochim. Biophys. Acta, Bioenerg. 253, 110 (1971).

${ }^{61}$ R. H. Sands and W. R. Dunham, Q. Rev. Biophys. 7(4), 443 (1975).

${ }^{62}$ J.-P. Gayda, P. Bertrand, C. More, and R. Cammack, Biochimie 63, 847 (1981).

${ }^{63}$ R. Malkin and A. J. Bearden, Proc. Natl. Acad. Sci. U. S. A. 68, 16 (1971).

${ }^{64}$ J. F. Gibson, D. O. Hall, J. H. M. Thornley, and F. R. Whatley, Proc. Natl. Acad. Sci. U. S. A. 56, 987 (1966).

${ }^{65}$ K. Mukai, T. Kimura, J. Helbert, and L. Kevan, Biochim. Biophys. Acta, Protein Struct. 295, 49 (1973).

${ }^{66}$ G. Palmer, R. H. Sands, and L. E. Mortenson, Biochim. Biophys. Res. Commun. 23, 357 (1966).

${ }^{67}$ D. A. Pantazis, W. Ames, N. Cox, W. Lubitz, and F. Neese, Angew. Chem., Int. Ed. 51, 9935 (2012).

${ }^{68}$ Y. Kurashige, G. K.-L. Chan, and T. Yanai, Nat. Chem. 5, 660 (2013).

${ }^{69}$ G. C. Dismukes and Y. Siderer, Proc. Natl. Acad. Sci. U. S. A. 78, 274 (1981).

${ }^{70}$ Ö. Hansson and L.-E. Andréasson, Biochim. Biophys. Acta, Bioenerg. 679, 261 (1982).

${ }^{71}$ D. J. Vinyard, S. Khan, M. Askerka, V. S. Batista, and G. W. Brudvig, J. Phys. Chem. B 121, 1020 (2017).

${ }^{72}$ B. O. Roos, R. Lindh, P.-Å. Malmqvist, V. Veryazov, and P.-O. Widmark, J. Phys. Chem. A 108, 2851 (2005).

${ }^{73}$ B. O. Roos, R. Lindh, P.-A. Malmqvist, V. Veryazov, and P.-O. Widmark, J. Phys. Chem. A 109, 6575-6579 (2005). 